\title{
Effects of Kinesio Taping on Balance and Gait in Patients with Stroke: A Systematic Review and Meta-Analysis of Studies in Korea
}

\author{
Byeong Geun Kim, Woon Su Cho, Yong Seong Kim \\ Department of Physical Therapy, Nambu University, Gwangju, Republic of Korea
}

\begin{abstract}
Purpose: The objective of this study was to investigate the effects of Kinesio taping (KT) for balance and gait in patients with stroke through meta-analysis of studies conducted in Korea.

Methods: RISS, Science on, and DBPia were the three databases used to collect articles on KT. Keywords such as "Stroke," "Kinesio taping," "Elasticity taping," and "Taping" were used to search for published articles. We systematically searched from the inception of each database to November 2020. Interventions and comparisons were KT and without KT. Outcome measures were the timed up and go (TUG) and 10-meter walking tests (10MWT). Consequently, six studies were selected for the second screening using meta-analyses.

Results: Based on the results of the meta-analysis, comparison between patients with and without KT showed that KT was effective for TUG (ES: 2.51, 95\% Cl: 2.12 to 2.90); however, it was not effective for 10MWT (ES: 0.79, 95\% Cl: -0.04 to 1.62 ).

Conclusion: The current evidence suggests that $\mathrm{KT}$ is more effective than without $\mathrm{KT}$ interventions for balance function in post-stroke patients. However, more well-conducted randomized controlled trials are required in the future.
\end{abstract}

Keywords: Stroke, Kinesio taping, Balance, Gait

\section{서 론}

뇌졸중은 건강부분에서 전 세계적으로 관심을 가지는 문제적 요소 이다.1.2 뇌졸중은 중년 및 노인들 사이에서 높은 사망률을 나타내며 지속적인 장애를 초래한다.-5 선행연구에 따르면 뇌졸중이 발생하고 생존한 사람의 약 $80 \%$ 가 기능적인 측면에서 장애를 겪게된다. ${ }^{67}$ 그러 므로 뇌졸중은 국가적으로 부담스러운 질병이다. ${ }^{8}$ 뇌졸중 환자는 여 러장애 중 자세 및 균형 조절 장애를 경험하게 된다.910 선행연구에 따 르면 뇌졸중 환자는 자세 및 균형 조절을 하기 위해 과도한 시각적 정 보에 의존한다 하였고, ${ }^{11}$ 우측 편마비 환자보다 좌측 편마비 환자가 자세 및 균형 조절의 어려움이 나타나며, ${ }^{12}$ 비대칭적 자세를 유지하는 데 있어 정상인보다 뇌졸중 환자가 자세 및 균형 조절이 어렵다고 보 고하였다.13 이러한 뇌졸중 환자의 자세 및 균형 장애는 낙상 위험을 증가시키고, ${ }^{14}$ 보행 능력에도 영향을 미치는 것으로 나타난다. ${ }^{1516}$ 선행 연구에 따르면 뇌졸중 환자의 $50 \%$ 이상이 지역사회에서 독립적인 보 행이 어렵다.1718 그러므로 뇌졸중 환자의 균형과 보행을 개선하는 것

Received Jan 12, 2021 Revised Feb 04, 2021

Accepted Feb 25, 2021

Corresponding author Yong Seong Kim

E-mail kys2492@naver.com

\section{은 중요한 과제이다.}

뇌졸중 환자의 기능 회복에 치료적 운동은 중요하다. ${ }^{19}$ 최근 뇌졸 중 환자에게 치료적 운동 중재 시 발목에 테이핑을 함께 적용한 연구 에서 균형과 보행 개선에 효과적이라고 보고되었다. 20,21 키네시오 테 이핑은 1970년대에 개발되어 전 세계적으로 보급되었다. 키네시오 테 이핑은 피부 및 피하 조직을 끌어당겨 혈액 순환을 촉진하고, 염증과 통증을 감소시키며, 신경근 재교육을 개선시키며, 부상을 예방하고 회복하는데 효과적이라고 알려져 있다.22 그러므로 국내에서도 뇌졸 중 환자 치료에 있어 키네시오 테이핑이 활발히 사용되고 있다.23-25 그 러나 국내에 뇌졸중 환자를 대상으로 진행된 연구가 많이 보고되지 만, 연구결과에 대해 종합적으로 효과크기를 계산하는 메타분석 연 구는 부족한 실정이다.

뇌졸중의 원인 중 하나인 뇌출혈 같은 경우, 서양인과 동양인 발병 비율도 다르고, ${ }^{26,27}$ 연구대상자의 신체조건이 차이가 나타난다. 현재 국외에 보고된 뇌졸중 환자에게 키네시오 테이핑 효과를 분석한 메 타분석 및 체계적 문헌고찰 연구에 국내 데이터베이스가 포함되어
Copylight (C)2021 The Korean Society of Physical Therapy

This is an Open Access article distribute under the terms of the Creative Commons Attribution Non-commercial License (https:// creativecommons.org/license/by-nc/4.0.) which permits unrestricted non-commercial use, distribution, and reproduction in any medium, provided the original work is properly cited. 
있지 않았다.28,29 국내에 보고된 뇌졸중 환자에게 테이핑의 효과를 문 헌 고찰한 연구에서는 효과크기를 계산하지 않은 한계가 있다. ${ }^{30}$ 그 러므로 국내 연구를 중심으로 한 연구가 필요하다. 따라서 본 연구의 목적은 국내에 발표된 뇌졸중 환자의 균형과 보행에 대해 발목 키네 시오 테이핑의 유효성과 안전성을 조사하기 위해 무작위 대조 연구 의 체계적인 검토 및 메타분석을 실시하였다.

\section{연구 방법}

\section{1. 문헌검색}

본 연구의 문헌검색은 한국교육학술정보원(RISS), 한국과학기술정보 연구원(Science on), 그리고 누리미디어(DBpia)의 데이터베이스에서 검색이 가능한 날부터 2020년 11월 30일까지의 문헌을 검색하였다. 검 색어는 '뇌졸중, '테이핑', '키네시오 테이핑', 그리고 '탄력 테이핑'을 사 용하였고, 한국어와 영어로 검색하였다. 데이터베이스 외의 사이트에 서 직접 검색은 실시하지 않았다.

\section{2. 선정기준}

본 연구의 대상자(P: participants)는 뇌졸중 진단 기준에 따라 컴퓨터 단층 촬영(CT) 또는 자기공명영상(MRI)으로 확인된 뇌졸중 환자이 다. 중재방법(I: intervention)은 물리치료와 함께 키네시오 테이핑을 중재한 방법이다. 비교 중재방법(C: comparison)은 물리치료 중재 시 테이핑을 중재하지 않거나, 가짜 테이핑을 적용한 중재이다. 평가 평 가(O: outcomes)는 국내 연구에서 많이 사용된 의자에서 일어나 걷기 검사와 $10 \mathrm{~m}$ 보행 검사를 선정하였다. 연구의 디자인(S: study design) 은 무작위 대조 연구를 선정하였다.

\section{3. 제외기준}

본 연구와 무관한 연구, 원문을 확인하지 못한 연구, 물리치료를 하지 않고 테이핑만 적용된 연구, 무작위 대조 연구가 아닌 연구는 제외하 였다.

\section{4. 연구선택}

본 연구에서 연구선택 시 서지관리 프로그램(Endnote X8)을 사용하 여 중복된 연구를 삭제하였다. 두 명의 연구자(1저자와 교신저자)가 선정기준 및 제외기준에 따라 제목과 초록을 독립적으로 검토하였 다. 검토된 연구는 추가로 두 명의 연구자(1저자와 교신저자)가 교차 로 검토하였다. 검토 시 불일치한 연구는 공동연구자와 토론으로 해 결하였다.

\section{5. 데이터 추출}

본 연구에서는 사전에 데이터 추출 양식을 엑셀프로그램에 설계하 였다. 선정된 연구에서 저자, 출판년도, 연구방법, 결과를 두 명의 연 구자가 독립적으로 추출하였다. 추출된 데이터는 추가로 두 명의 연 구자(1저자와 교신저자)가 교차로 검토하였다. 검토 시 불일치한 데이 터는 공동연구자와 토론으로 해결하였다.

\section{6. 비뚤림 위험 평가}

본 연구에 선정된 무작위 대조 연구에 대한 방법론적 질 평가는 Cochrane의 Risk of Bias (RoB) 기준에 따라 두 명의 연구자(1저자와 교신 저자)가 독립적으로 평가하였다. 비뚤림 위험 평가에는 무작위 배정 순서 생성, 배정순서 은폐, 연구참여자 그리고 연구자에 대한 눈가림, 결과평가에 대한 눈가림, 불충분한 결과자료, 선택적 보고, 그 외 비 뚤림이 포함되어 있다. 평가 후 두 명의 연구자(1저자와 교신저자)가 교차로 검토하였다. 검토 시 불일치한 평가는 공동연구자와 토론으 로 해결하였다.

\section{7. 통계분석}

본 연구에서 메타분석은 Revman 5.4 를 사용하였다. 선정된 연구의 대상자 수의 효과크기 분포 평균을 추정하기 위해 무선효과모형 (random effects model)을 사용하였다. 본 연구에서 선정된 연구들이 동일한 단위를 사용한 의자에서 일어나 걷기 검사는 평균 차이(mean difference, $\mathrm{MD}$ )를 사용하여 평균 효과 크기를 산출하였고, 동일한 단 위를 사용하지 않은 $10 \mathrm{~m}$ 보행 검사는 표준화 평균차이(standardized mean difference, $\mathrm{SMD}$ )를 사용하여 평균 효과 크기를 산출하였다. 중 재 기간에 따라 하위그룹분석을 하였다. 선정된 연구 간에 이질성을 분석하기 위해 전체 분산을 산출하기 위하여 카이제곱 검정을 사용 하였고, 추가로 연구 간 분산 비율을 확인하기 위하여 $\mathrm{I}^{2}$ 값을 산출하 였다. 효과크기에 대한 이질성은 유의확률이 0.10 이상이고, $\mathrm{I}^{2}$ 가 $50 \%$ 이하인 경우 낮은 이질성으로 간주하며, 유의확률이 0.10 이하이고, $\mathrm{I}^{2}$ 가 $50 \%$ 이상인 경우 높은 이질성으로 간주한다. 이질성이 확인된 경 우 민감도 분석을 적용하였다.

\section{결 과}

\section{1. 연구 선택}

본 연구에서 관련 검색어를 사용해서 검색한 결과 총 524 편의 연구가 검색되었다. 중복 제거 후 연구의 제목과 초록을 검토한 결과, 본 연구 와 관련이 없는 75 편의 연구가 제외 되었다. 25 편의 연구의 원문을 확 인한 결과 19 편이 제외되고, 최종 6 편의 연구가 본 연구에 포함되었 다.31-36 선정된 연구 선택의 절차는 그림과 같다(Figure 1). 


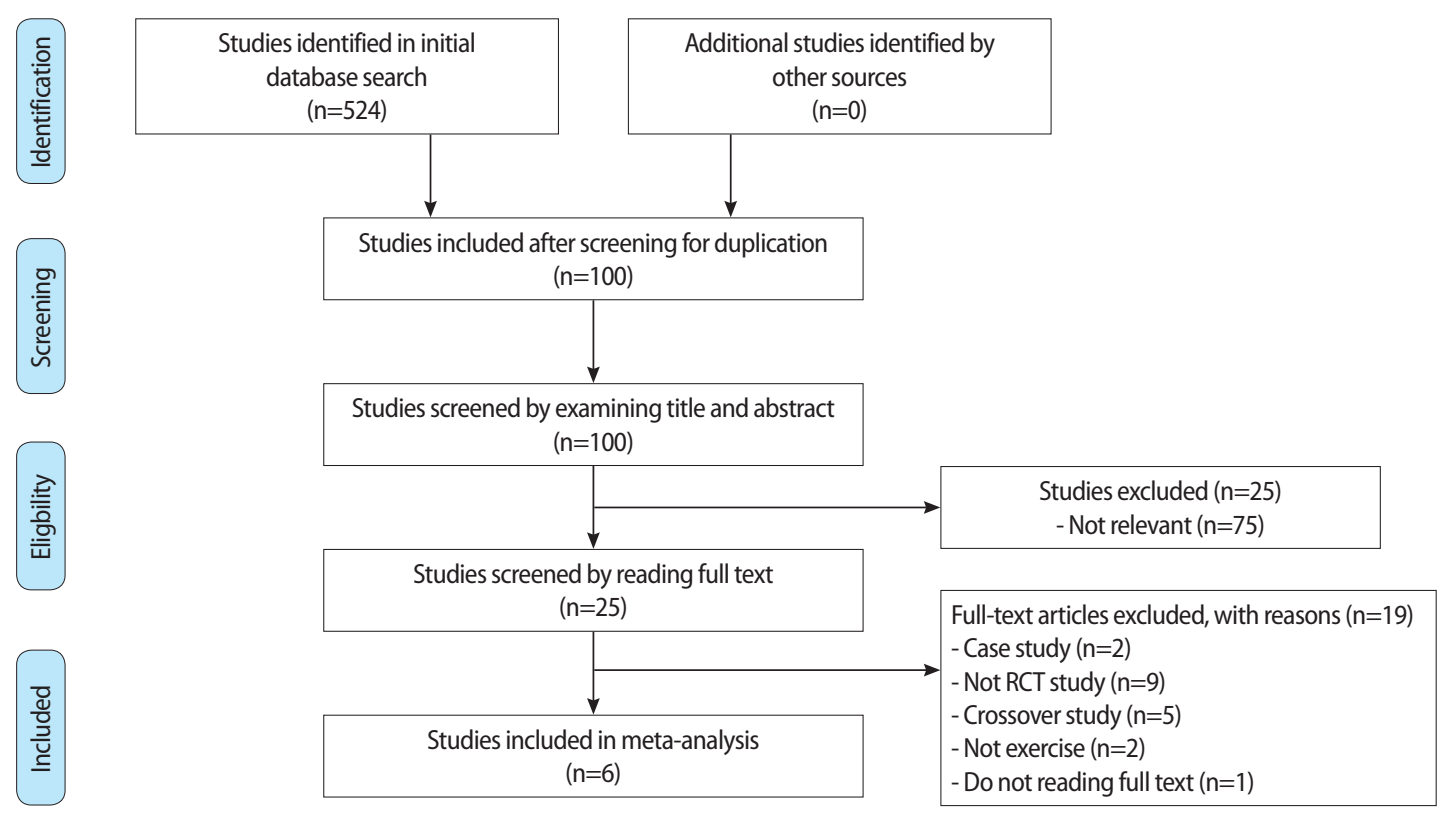

Figure 1. PRISMA flow diagram of systematic review study selection process.

Table 1. Characteristics of studies included in the analysis

\begin{tabular}{|c|c|c|c|c|}
\hline Study (type) & Participants & Intervention & Duration of intervention & $\begin{array}{l}\text { Outcome } \\
\text { measure }\end{array}$ \\
\hline $\begin{array}{l}\text { Kim et al., } 2012 \\
\text { (Journal) }\end{array}$ & $\begin{array}{l}\text { Total sample size 26(G1: } 13 \text {, male: 5, female: 8; G2: 13, } \\
\text { male: } 4 \text {, female: } 9 \text { ), Mean age (SD): } G 1=58.85(11.26) \text {; } \\
\text { G2=58.77(14.46), Stroke onset: no }\end{array}$ & G1: KT (TAT, GT, 8T)+TEP, G2: TEP & $\begin{array}{l}\text { Once } 30 \text { minutes, } 3 \text { times a } \\
\text { week, } 8 \text { weeks in total }\end{array}$ & TUG, 10MWT \\
\hline $\begin{array}{l}\text { Jeong et al., } 2016 \\
\text { (Journal) }\end{array}$ & $\begin{array}{l}\text { Total sample size } 20 \text { (G1: } 10 \text {, male: } 5 \text {, female: } 5 ; \mathrm{G} 2: 10 \text {, } \\
\text { male: } 5 \text {, female: } 5) \text {, Mean age (SD): } \mathrm{G} 1=61.20(7.10) \text {; } \\
\text { G2=62.10(8.13), Stroke onset: } 12 \sim 24 \text { months }\end{array}$ & $\begin{array}{l}\text { G1: KT (EHT, EDT, TAT)+Treadmill, } \\
\text { G2: Treadmill }\end{array}$ & $\begin{array}{l}\text { Once } 50 \text { minutes, } 5 \text { times a } \\
\text { week, } 6 \text { weeks in total }\end{array}$ & TUG, 10MWT \\
\hline $\begin{array}{l}\text { No et al., } 2013 \\
\text { (Journal) }\end{array}$ & $\begin{array}{l}\text { Total sample size } 22 \text { (G1: } 7 \text {, male: } 3 \text {, female: } 4 ; \text { G2: } 7 \text {, } \\
\text { male: } 3 \text {, female: } 4 ; G 3: 8 \text {, male: } 6 \text {, female: } 2) \text {, Mean } \\
\text { age (SD): G1 }=68.00(7.48) ; G 2=62.85(3.67) \text {; } \\
\text { G3 }=61.25(7.26) \text {, Stroke onset: more than } 6 \text { months }\end{array}$ & $\begin{array}{l}\text { G1: KT (TAT)+FMS+Treadmill, } \\
\text { G2: KT (TAT)+Treadmill, } \\
\text { G3: Treadmill }\end{array}$ & $\begin{array}{l}\text { Once } 30 \text { minutes, } 3 \text { times a } \\
\text { week, } 6 \text { weeks in total }\end{array}$ & TUG, 10MWT \\
\hline $\begin{array}{l}\text { Park et al.., } 2017 \\
\quad \text { (Journal) }\end{array}$ & $\begin{array}{l}\text { Total sample size } 25(\mathrm{G} 1: 14 \text {, male: } 11 \text {, female: } 3 ; \mathrm{G} 2: 11 \text {, } \\
\text { male: } 6 \text {, female: } 5) \text {, Mean age (SD): } \mathrm{G} 1=60.43(12.92) \text {; } \\
\mathrm{G} 2=65.45(6.47) \text {, Stroke onset: more than } 6 \text { months }\end{array}$ & $\begin{array}{l}\mathrm{G} 1: \mathrm{KT}(\mathrm{GT})+\mathrm{BE}, \mathrm{G} 2 \text { : Placebo } \\
\text { taping+BE }\end{array}$ & $\begin{array}{l}\text { Once } 30 \text { minutes, } 5 \text { times a } \\
\text { week, } 2 \text { weeks in total }\end{array}$ & TUG \\
\hline $\begin{array}{l}\text { Seo, } 2017 \\
\text { (Thesis) }\end{array}$ & $\begin{array}{l}\text { Total sample size } 30 \text { (G1: } 15 \text {, male: } 9 \text {, female: } 6 ; G 2: 15 \text {, } \\
\text { male: } 10 \text {, female: } 5 \text { ), Mean age (SD): } G 1=54.60(9.56) \text {; } \\
\text { G2=54.47(8.18), Stroke onset: more than } 6 \text { months }\end{array}$ & $\begin{array}{l}\text { G1: KT (APT, ADT)+Treadmill, } \\
\text { G2: Treadmill }\end{array}$ & Once 30 minutes & TUG \\
\hline $\begin{array}{l}\text { Um et al., } 2019 \\
\text { (Journal) }\end{array}$ & $\begin{array}{l}\text { Total sample size } 30(G 1: 10 \text {, male: } 7 \text {, female: } 3 ; \mathrm{G} 2: 10 \text {, } \\
\text { male: } 6 \text {, female: } 4 ; \mathrm{G} 3: 10 \text {, male: } 8 \text {, female: } 2) \text {, Mean } \\
\text { age (SD): } \mathrm{G} 1=59.79(11.05) ; \mathrm{G} 2=59.30(13.16) \text {; } \\
\text { G3 }=51.10(15.00) \text {, Stroke onset: more than } 6 \text { months }\end{array}$ & $\begin{array}{l}\text { G1: KT (AJT)+NKT(HJ)+TEP, } \\
\text { G2: KT (AJT)+TEP, G3: TEP }\end{array}$ & $\begin{array}{l}\text { Once } 30 \text { minutes, } 5 \text { times a } \\
\text { week, } 4 \text { weeks in total }\end{array}$ & TUG \\
\hline
\end{tabular}

G: group, SD: standard deviation, KT: kinesio taping, TAT: tibialis anterior taping, GT: gastrocnemius taping, 8T: 8 taping, TEP: therapeutic exercise program, TUG: timed up and go, 10MWT: 10 meter walking test, EHT: extensor halluces muscle taping, EDT: extensor digitorum muscle taping, FMS: functional electrical stimulaon, BE: balance exercise, APT: ankle proximal taping, ADT: ankle distal taping, AJT: ankle joint taping, NKT: non-KT, HJ: hip joint.

\section{2. 연구 특성}

6 편의 무작위 대조 연구에서 153 명의 환자가 참여했다. 그 중 86 명은 환자가 실험군, 67 명의 환자가 대조군에 참여되었다. 선정된 연구는 2012년부터 2019년까지 발표된 연구이며, 그 중 학위논문이 1편과 학 술논문이 5편이다. 연구에 사용된 언어는 5편이 한국어, 1 편이 영어 로 되어있다. 선정된 연구의 세부 특성은 표와같다(Table 1).

\section{3. 방법론적 질 평가}

선정된 무작위 대조 연구에 대한 Cochrane Collaboration의 RoB 도구 로 평가된 비뚤림 위험 평가는 그림과 같다(Figure 2A, 2B). 무작위 배 정순서 생성에서 1 편의 연구만 적절하게 보고하였다. ${ }^{36}$ 배정순서 은 폐는 모든 연구에서 불확실하였다. ${ }^{31-36}$ 연구 참여자, 연구자, 그리고 결 과평가에 대한 눈가림에서는 모든 연구가 높은 비뚤림 위험으로 평 가되었다. ${ }^{31-36}$ 불충분한 결과자료에서 1 편의 연구에서 환자의 탈락율 

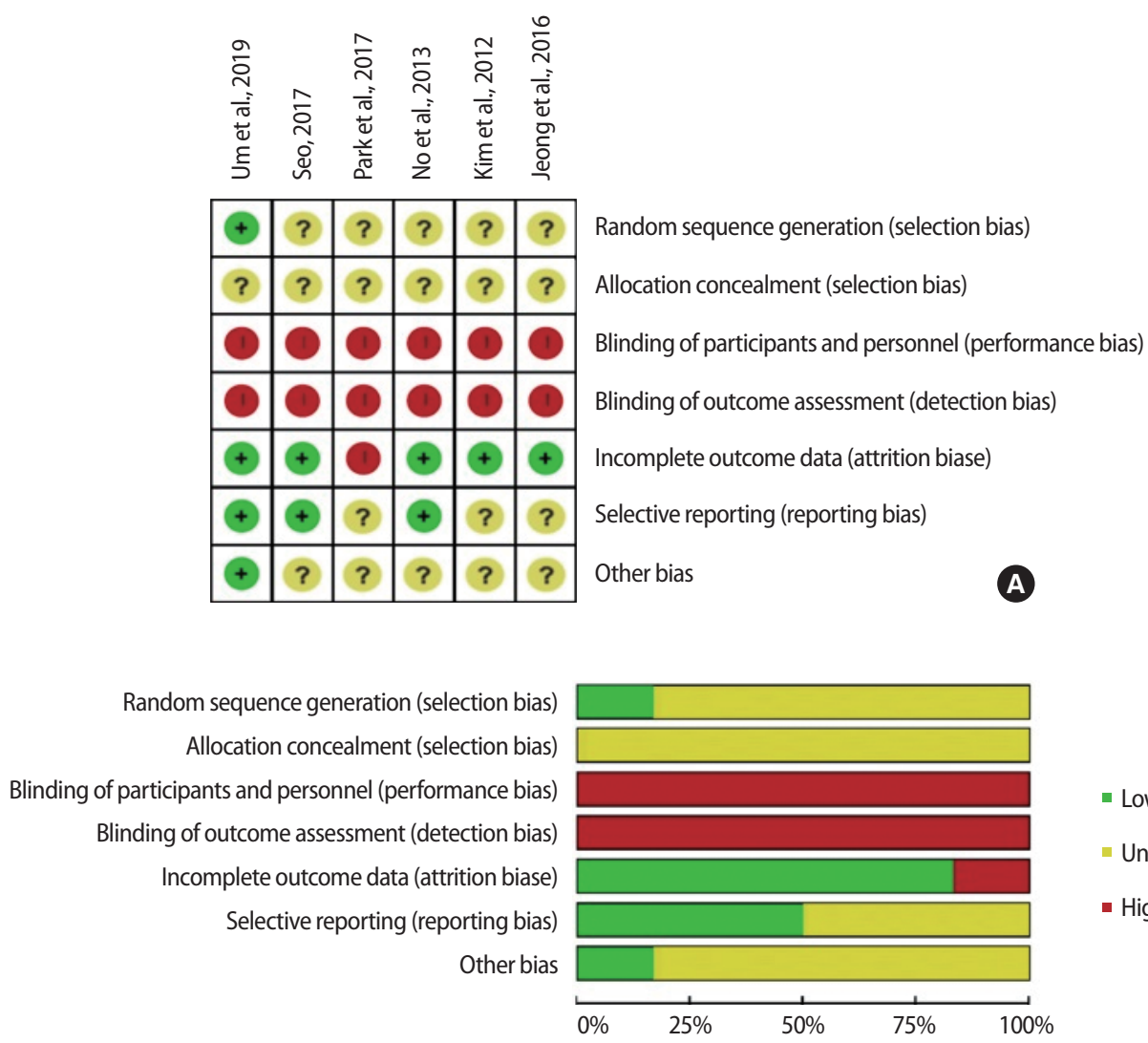

- Low risk of bias

- Unclear risk of bias

- High risk of bias

B

Figure 2. Methodological evaluation of RCT study using RoB tool.

이 $27 \%$ 로 높은 비뚤림 위험으로 평가되었다. ${ }^{34}$ 선택적 보고에서 3 편의 연구만 연구프로토콜을 기관생명윤리위원회의 승인을 받아서 낮은 비뚤림 위험으로 평가되었다. ${ }^{33,35,36}$ 그 외 비뚤림에서는 1 편의 연구만 자금 출처를 보고하여 낮은 비뚤림 위험으로 평가되었다. ${ }^{36}$

\section{4. 의자에서 일어나 걷기 검사에 대해 키네시오 테이핑 적용 유무 비교}

6편의 연구에서 물리치료 시 키네시오 테이핑 적용 유무를 비교하였 다. 의자에서 일어나 걷기 검사에서 키네시오 적용 유무 사이에 유의 한 차이가 있었다(MD =2.51, 95\%CI: 2.12 to 2.90, $\mathrm{p}<0.00001$ )(Figure 3). 중재 기간에 따라 하위그룹 분석을 실시하였다. 하위그룹 분석 결과 는 2 주에서 가장 큰 효과크기를 나타내었다. ${ }^{34} 6$ 편의 연구에서 이질성 은 $I^{2}=95 \%$ 로 높은 이질성이 있는 것으로 나타났다. 민감도 분석을 시행 한 결과 $\mathrm{Um}$ 등 ${ }^{36}$ 의 연구를 제거함으로써 $\mathrm{I}^{2}=95 \%>61 \%$ 로 감소하였다.

\section{5. $10 \mathrm{~m}$ 보행 검사에 대해 키네시오 테이핑 적용 유무 비교}

3편의 연구에서 물리치료 시 키네시오 테이핑 적용 유무를 비교하였다 $10 \mathrm{~m}$ 보행 검사에서 키네시오 유무 사이에 유의한차이가 없었다 $(\mathrm{SMD}=$ $0.79,95 \% \mathrm{CI}:-0.04$ to $1.62, \mathrm{p}=0.06$ )(Figure 4 ).

\section{고 찰}

본 연구는 국내에서 뇌졸중 환자의 발목에 키네시오 테이핑을 중재 하여 균형과 보행에 대해 분석한 첫 번째 메타분석 연구이다. 본 연구 의 결과, 뇌졸중 환자의 균형에서 물리치료 시 발목 키네시오 테이핑 중재는 키네시오 테이핑을 중재하지 않고 물리치료를 하는 것 보다 더 효과가 있는 것으로 나타났다. 본 연구에서 중재 기간에 따라 하위 그룹 분석을 실시한 결과, 균형은 2주 이상부터 효과가 있었고, 보행 은 8주이상 부터 효과가 있는 것으로 나타났다.

본 연구의 균형 결과는 뇌졸중 환자에게 물리치료 시 키네이오 테 이핑을 적용했을 때 기능적 회복과 장점을 보고한 선행연구들과 유 사한 결과를 나타내었다. 29,37,38

본 연구에서 키네시오 테이핑 중재는 발목에만 중재한 연구를 중 심으로 메타분석을 실시하였다. 발목은 균형을 유지하기 위해서 가 장 먼저 사용되는 발목 전략을 사용하는 관절이다. ${ }^{39}$ 키네시오 테이핑 은 지속적인 근육수축을 유도하고 림프 및 혈액 순환을 개선한다. ${ }^{40}$ 그 러므로 물리치료 시 뇌졸중 환자의 마비측 다리에 키네시오 테이핑을 적용하는 것은 지속적인 근육 수축을 유도하므로 효과적으로 작용 한 것으로 사료된다. 그래서 균형 평가에서 발목 전략이 효과적으로 


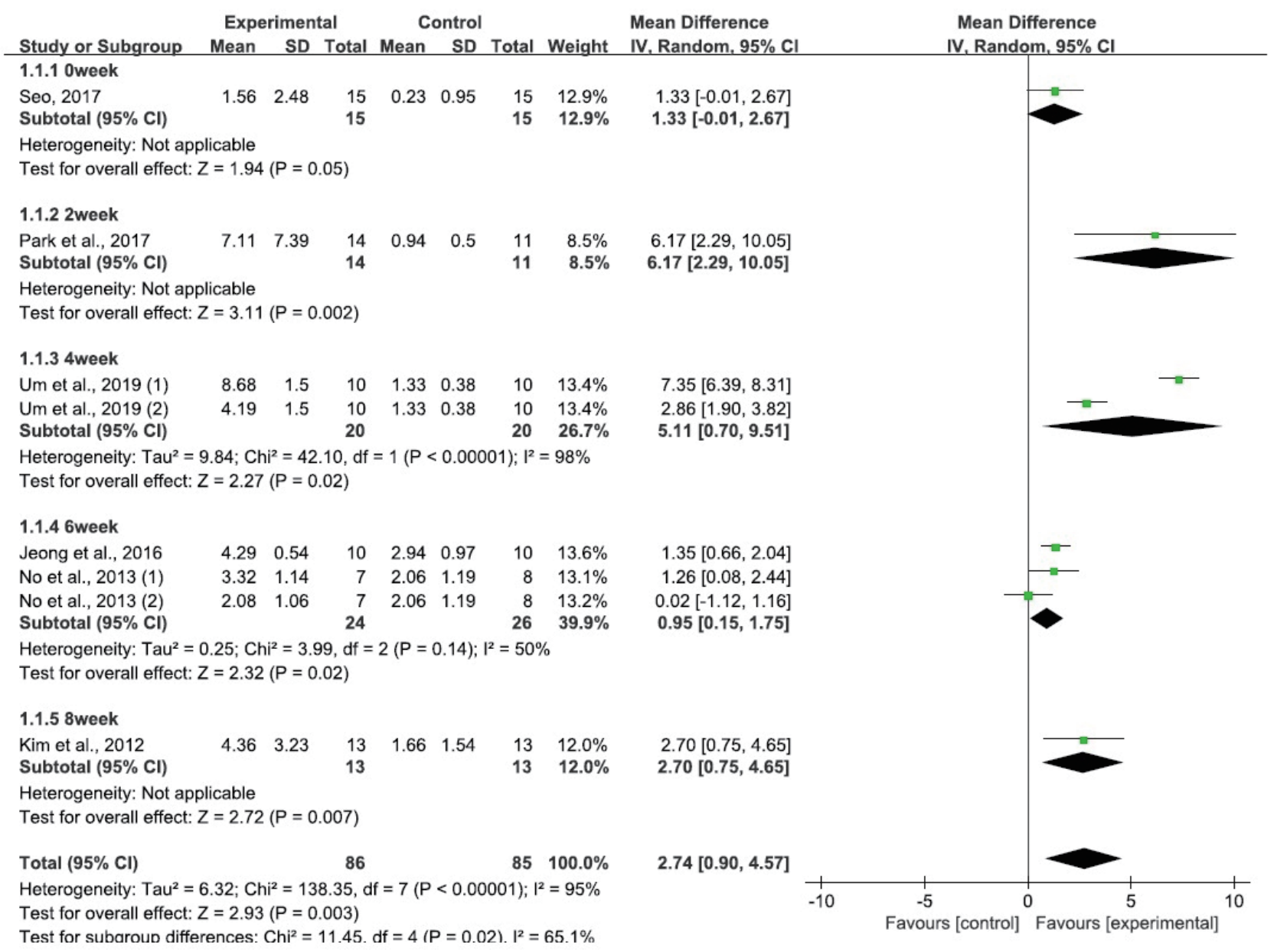

Figure 3. Meta-analysis of difference in TUG between groups after intervention.

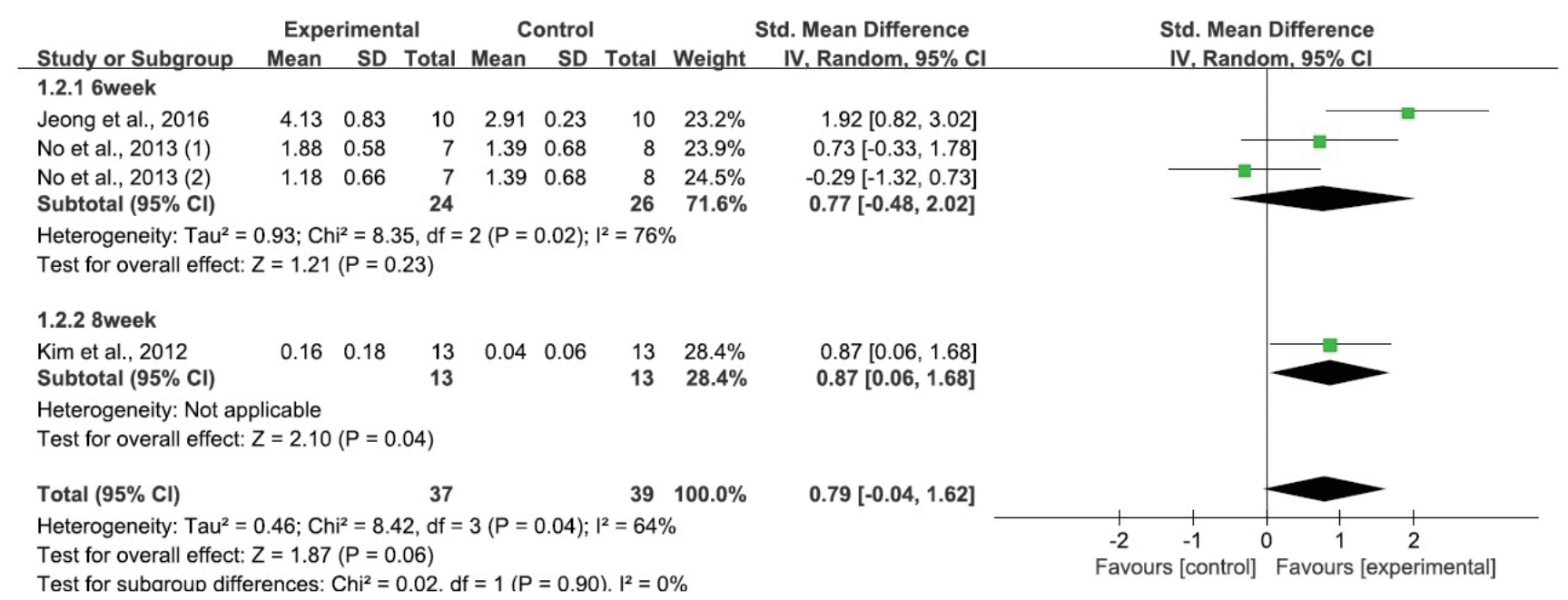

Figure 4. Meta-analysis of difference in 10MWT between groups after intervention.

작용한 것으로 생각된다.

본 연구의 보행 결과는 물리치료 시 발목 키네시오 테이핑 중재는 키네시오 테이핑을 중재하지 않고 물리치료를 했을 때와 유의한 차
이가 나타나지 않았다. 이러한 결과는 국외의 메타분석 선행연구의 결과와 일치하였다. ${ }^{28}$

정상적인 보행은 다리의 움직임뿐만 아니라 머리, 몸통, 그리고 팔 
까지 보행에 영향을 미친다. ${ }^{41}$ 뇌졸중 환자의 손상된 몸통과 팔은 보 행에 영향을 미친다고 보고되었다. ${ }^{42-44}$ 그러므로 보행은 전신이 사용 되는 관점을 고려해야한다. 본 연구의 결과와 같이 뇌졸중 환자에게 키네시오 테이핑을 발목에만 적용하는 것은 효과적이지 않다고 생각 된다.

본 연구에서 중재기간에 따른 분석은 4 주 이상의 중재 시 균형이 효과가 있다는 선행연구, ${ }^{28} 4$ 주 중재가 6주 중재 보다 효과적이라는 또 다른 선행연구와도 결과가 일치하지 않았다. ${ }^{45}$ 중재기간에 따른 효 과는 선행연구들과 본 연구 결과는 모두 상반대 결과를 나타내고 있 다. 이러한 결과는 중재기간 마다 포함된 연구 수가 적어 이질성이 나 타났기 때문이라고 생각된다. 임상에서 추가적으로 연구가 진행되어 이질성에 대한 분석이 필요하다.

본 연구를 통해 뇌졸중 환자의 균형에 대한 키네시오 테이핑의 최 신 근거를 제공했다. 키네시오 테이핑은 임상에서 뇌졸중 환자의 다 양한 중재 방법과 함께 사용될 수 있음을 확인하였다. 본 연구에는 몇 가지 제한점이 있다. 첫째, 선정된 연구의 수가 적었다. 둘째, 연구 에서 중재한 기간이 즉각적인 연구부터 8 주까지 일괄적이지 않았다. 셋째, 키네시오 테이핑 적용을 발목으로 한정하였지만 발목에서 테 이핑 적용 방법이 다양했다. 따라서 향후에 뇌졸중 환자의 발목에 표 준화된 키네시오 테이핑을 적용하기 위해서는 질 높은 연구방법론 그리고 많은 표본의 연구들이 필요하다.

결론적으로, 본 연구는 현재까지 연구된 근거를 배경으로 물리치 료 시 키네시오 테이핑 중재가 물리치료만 중재 하거나, 가짜 테이핑 을 적용한 중재 보다 뇌졸중 환자의 균형 능력에 더 효과적임을 입증 했다. 효과적인 중재기간에 대해서는 추가적인 무작위 대조연구가 필 요하다.

\section{REFERENCES}

1. Feigin VL, Nguyen G, Cercy K et al. Global, regional, and country-specific lifetime risks of stroke, 1990 and 2016. N Engl J Med. 2018;379 (25):2429-37.

2. Murray CJL, Barber RM, Foreman KJ et al. Global, regional, and national disability-adjusted life years (DALYs) for 306 diseases and injuries and healthy life expectancy (HALE) for 188 countries, 1990-2013: quantifying the epidemiological transition. Lancet. 2015;386(10009):2145-91.

3. Roth EJ, Lovell L. Community skill performance and its association with the ability to perform everyday tasks by stroke survivors one year following rehabilitation discharge. Top Stroke Rehabil. 2007;14(1):48-56.

4. Guan T, Ma J, Li M et al. Rapid transitions in the epidemiology of stroke and its risk factors in china from 2002 to 2013. Neurology. 2017;89(1): 53-61.

5. Zhang Y, Wang S, Chen P et al. Tai chi for stroke rehabilitation: protocol for a systematic review. BMJ Open. 2016;6(6):e010866.

6. Zheng G, Huang M, Liu F et al. Tai chi chuan for the primary prevention of stroke in middle-aged and elderly adults: a systematic review. Evid Based Complement Alternat Med. 2015;2015:742152.

7. Chen BL, Guo JB, Liu MS et al. Effect of traditional chinese exercise on gait and balance for stroke: a systematic review and meta-analysis. PLoS One. 2015;10(8):e0135932.

8. Krishnamurthi RV, Ikeda T, Feigin VL. Global, regional and countryspecific burden of ischaemic stroke, intracerebral haemorrhage and subarachnoid haemorrhage: a systematic analysis of the global burden of disease study 2017. Neuroepidemiology. 2020;54(2):171-9.

9. Tyson SF, Hanley M, Chillala J et al. Balance disability after stroke. Phys Ther. 2006;86(1):30-8.

10. Perennou DA, Mazibrada G, Chauvineau V et al. Lateropulsion, pushing and verticality perception in hemisphere stroke: a causal relationship? Brain. 2008;131(9):2401-13.

11. Bonan IV, Colle FM, Guichard JP et al. Reliance on visual information after stroke. part I: balance on dynamic posturography. Arch Phys Med Rehabil. 2004;85(2):268-73.

12. Rode G, Tiliket C, Boisson D. Predominance of postural imbalance in left hemiparetic patients. Scand J Rehabil Med. 1997;29(1):11-6.

13. Genthon N, Rougier P, Gissot AS et al. Contribution of each lower limb to upright standing in stroke patients. Stroke. 2008;39(6):1793-9.

14. Xu T, Clemson L, O'Loughlin K et al. Risk factors for falls in community stroke survivors: A systematic review and meta-analysis. Arch Phys Med Rehabil. 2018;99(3):563-73.

15. Van de Port I, Kwakkel G, Lindeman E. Community ambulation in patients with chronic stroke: how is it related to gait speed? J Rehabil Med. 2008;40(1):23-7.

16. Durcan S, Flavin E, Horgan F. Factors associated with community ambulation in chronic stroke. Disabil Rehabil. 2016;38(3):245-9.

17. Blennerhassett JM, Levy CE, Mackintosh A et al. One-quarter of people leave inpatient stroke rehabilitation with physical capacity for community ambulation. J Stroke Cerebrovasc Dis. 2018;27(12):3404-10.

18. Perry J, Garrett M, Gronley JK et al. Classification of walking handicap in the stroke population. Stroke. 1995;26(6):982-9.

19. Ramos-Lima MJM, Brasileiro IdC, Lima TLd et al. Quality of life after stroke: impact of clinical and sociodemographic factors. Clinics. 2018; 73:e418.

20. Park SJ, Kim TH, Oh SH. Immediate effects of tibialis anterior and calf muscle taping on center of pressure excursion in chronic stroke patients: a cross-over study. Int J Environ Res Public Health. 2020;17(11):4109.

21. Sheng Y, Kan S, Wen Z et al. Effect of kinesio taping on the walking ability of patients with foot drop after stroke. Evid Based Complement Alternat Med. 2019;2019:2459852.

22. Celiker R, Guven Z, Aydog T et al. The kinesiologic taping technique and its applications/kinezyolojik bantloma teknigi ve uygulama alanlari. Turk J Phys Med Rehabil. 2011;57(4):225-36.

23. Park SJ. Effect of inspiratory muscle training with elastic taping on forced vital capacity and sway area in stroke patients. J Kor Phys Ther. 2020;32 (2):121-5.

24. Park DW, Kang TW, Lee KS. Effects of ankle mobilization with movement taping on ankle range of motion and gait functions in patients with chronic stroke with limited ankle dorsiflexion. Kor J Neuromuscul Rehabil. 2020;10(2):48-56.

25. Kim HW, Park YH. Effects of incorporating non-elastic Taping into PNF 
techniques on muscle activities, balance, and gait in patients with chronic stroke. J Korean Soc Phys Med. 2020;15(1):113-21.

26. Liao HC, Chen SH, Yang CD et al. Clinical profile and outcomes of early seizures in asian patients with acute intracerebral hemorrhage. J Acute Med. 2019;9(4):172-7.

27. Yakushiji Y, Tanaka J, Wilson D et al. Proportion of intracerebral haemorrhage due to cerebral amyloid angiopathy in the east and west: Comparison between single hospital centres in Japan and the united kingdom. J Neurol Sci. 2020;416:117037.

28. Hu Y, Zhong D, Xiao Q et al. Kinesio taping for balance function after stroke: a systematic review and meta-analysis. Evid Based Complement Alternat Med. 2019;2019:8470235.

29. Wang M, Pei ZW, Xiong BD et al. Use of kinesio taping in lower-extremity rehabilitation of post-stroke patients: a systematic review and metaanalysis. Complement Ther Clin Pract. 2019;35:22-32.

30. Kim BR, Kang TW. A systematic review of elastic taping effect of patients with stroke. J Kor Phys Ther. 2018;30(4):101-7.

31. Kim YR, Kim JI, Kim YY et al. Effects of ankle joint taping on postural balance control in stroke patients. J Int Acad Phys Ther Res. 2012;3(2): 413-78.

32. Jeong WM, Kim BR, Kang MG. Effect of treadmill training and proprioceptive neuromuscular facilitation lower leg taping on balance and gait ability in stroke patients. PNF \& Mov. 2016;14(2):83-91.

33. Noh KS, Hur JG, Ko JY. The effects of gait training with lower limb taping combined with functional electrical stimulation on stroke patient's muscle activity and gaits. J Korean Acad Ther. 2013;5(2):37-49.

34. Park SJ, Kim TH, Go JH et al. The impact of convergence balance training and taping on spasticity and balance ability in patients with chronic stroke. J Digit Converg. 2017;15(7):297-306.

35. Seo MY. Immediate effects of ankle pronation taping on balance and gait in patients with chronic stroke. Daegu University. Dissertation of Master' s Degree. 2017.

36. Um YJ, Jang HY, Lee SM. Taping therapy simultaneously applied to the ankle and hip joint: effect on balance and gait in patients with chronic stroke. J Kor Phys Ther. 2019;31(1):1-7.

37. Ortiz-Ramirez J, Perez-De la Cruz S. Efficacy of the application of kinesio tape in patients with stroke. Rev Neurol. 2017;64(4):175-9.

38. Munoz-Barrenechea IA, Garrido-Beroiza MA, Achiardi O et al. A systematic review of the functional effectiveness of kinesio taping in individuals with ankle instability. Medwave. 2019;19(4):e7635.

39. Shumway-Cook A, Woollacott M. Attentional demands and postural control: the effect of sensory context. J Gerontol A Biol Sci Med Sci. 2000;55(1):M10-6.

40. Boeskov B, Carver LT, Essen-Leise AV et al. Kinesthetic taping improves walking function in patients with stroke: a pilot cohort study. Top Stroke Rehhanil. 2014;21(6):495-501.

41. Perry J, Burnfield JM. Gait analysis : normal and pathological function. 2th ed. California, Slack, 2010:1-556.

42. Balaban B, Tok F. Gait disturbances in patients with stroke. PM\&R. 2014;6(7):635-42.

43. Van Criekinge T, Saeys W, Hallemans A et al. Trunk biomechanics during hemiplegic gait after stroke: a systematic review. Gait Posture. 2017; 54:133-43.

44. Frykberg GE, Johansson GM, Schelin L et al. The arm posture score for assessing arm swing during gait: an evaluation of adding rotational components and the effect of different gait speeds. Gait Posture. 2014;40(1): 64-9.

45. Yanhua W, Zhuangmiao L, Xu D. Effects of kinesio taping at different intervention time on the motor function of lower extremity after stroke: a meta-analysis. CRTER. 2018;22(32):5241-8. 